\title{
Fuzzy 3D Face Ethnicity Categorization
}

\author{
Cheng Zhong, Zhenan Sun, and Tieniu Tan \\ National Laboratory of Pattern Recognition, Institute of Automation, \\ Chinese Academy of Sciences, Beijing, 100080, P.R. China \\ \{czhong, znsun, tnt\}@nlpr.ia.ac.cn
}

\begin{abstract}
In this paper, we propose a novel fuzzy 3D face ethnicity categorization algorithm, which contains two stages, learning and mapping. In learning stage, the visual codes are first learned for both the eastern and western individuals using the learned visual codebook (LVC) method, then from these codes we can learn two distance measures, merging distance and mapping distance. Using the merging distance, we can learn the eastern, western and human codes based on the visual codes. In mapping stage, we compute the probabilities for each 3D face mapped to eastern and western individuals using the mapping distance. And the membership degree is determined by our defined membership function. The main contribution of this paper is that we view ethnicity categorization as a fuzzy problem and give an effective solution to assign the 3D face a reasonable membership degree. All experiments are based on the challenging FRGC2.0 3D Face Database. Experimental results illustrate the efficiency and accuracy of our fuzzy 3D face ethnicity categorization method.
\end{abstract}

Keywords: 3D Face, Fuzzy Ethnicity Categorization, Learned Visual Codebook.

\section{Introduction}

Automatic identification of human faces is a very challenging research topic, which has gained much attention during the last few years [1. However, human faces can not only provide the identities, but also the demographic information, such as gender and ethnicity, which are also necessary for human face perception.

Some effort has been made on the ethnicity categorization area over the last two decades. Gutta et al. 2] treated ethnicity categorization as a multi-class problem including Caucasian, Asian, Oriental and African origins categories. Based on the intensity faces, they adopted a hybrid classifier, ensemble of radial basis functions and a decision tree, for the categorization. Qiu et al. 3. considered ethnicity categorization as a two-class problem, including asian and non-asian. Based on the iris image, they combined the Gabor features and the Adaboost classifier to achieve categorization. Lu et al. 4] also viewed ethnicity categorization as a two-class problem. In their solution, they adopt SVM for both the intensity and depth modalities, and the matching scores were combined 


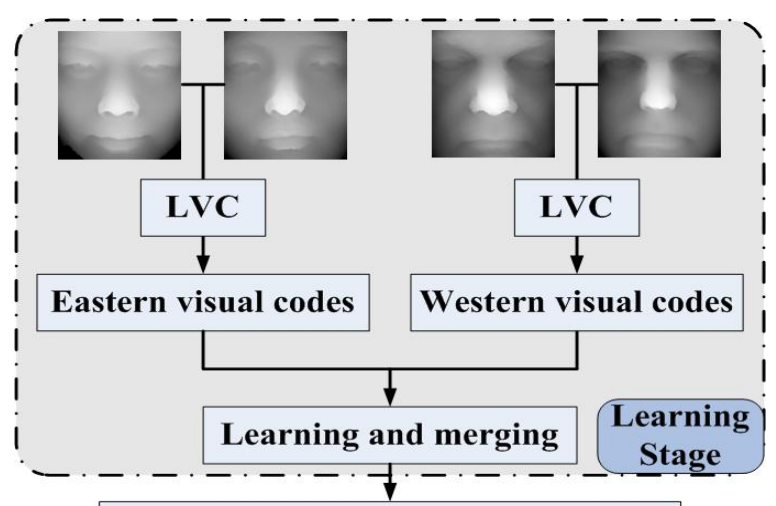

Eastern, western and human codes

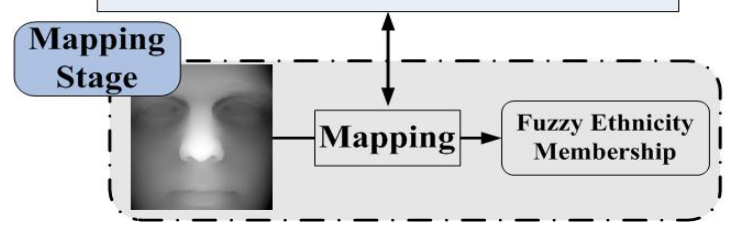

Fig. 1. The flowchart of our fuzzy 3D face categorization

using the sum rule. The drawback of these works [3] [4] is that the ethnicity categorization is not a simple two-class problem (asian and non-asian), and there are also some other ethnic categories, such as Latin and Indian. Although [2] proposed four ethnic categories, it can not cover all the ethnic groups.

In this paper, we propose a fuzzy $3 \mathrm{D}$ face ethnicity categorization algorithm to solve the ethnic problems. The flowchart is shown in Fig 1, In learning stage, we first learn the eastern and western visual codes using the training eastern and western 3D faces based on the LVC method [5], then the merging and mapping distances are learned based on all the visual codes. When the distance between two different visual codes (eastern and western) is below merging distance, they are assigned to the human codes, which are viewed as the common properties of humans. The remaining eastern and western visual codes become the eastern and western codes (the specific properties of eastern and western individuals). In mapping stage, for each 3D face, we compute its probabilities for both eastern and western using the mapping distance and determine its membership degree by our membership function.

The main contribution of this paper is the introduce of fuzzy membership degree into the ethnicity categorization. Therefore, we need not to pay much attention on whether we can cover all of the ethnic groups. And how to accurately extract the ethnic information embedded in the 3D faces becomes our main task.

The remainer of this paper is organized as follows. In Section 2, we give the detail information of our fuzzy 3D face categorization. We describe our experimental results and discussions in Section 3. Finally, this paper is concluded in Section 4 . 


\section{Fuzzy 3D Face Categorization}

\subsection{A Brief Introduction of LVC}

In [5], Zhong introduced the Learned Visual Codebook (LVC) into 3D face recognition. This method can be divided into two stages, training and mapping. In training stage, they first extracted intrinsic discriminative information embedded in 3D faces using Gabor filters, then K-means clustering was adopted to learn the centers from the filter response vectors, which can be viewed as the visual codes. In mapping stage, they compared Gabor feature vectors with the learned codes. And each Gabor feature vector was mapped to the learned code with the nearest distance. A mapping histogram was constructed to represent each 3D face. The flowchart of LVC is shown in Fig,2 For detailed information of this algorithm, you can refer to [5].

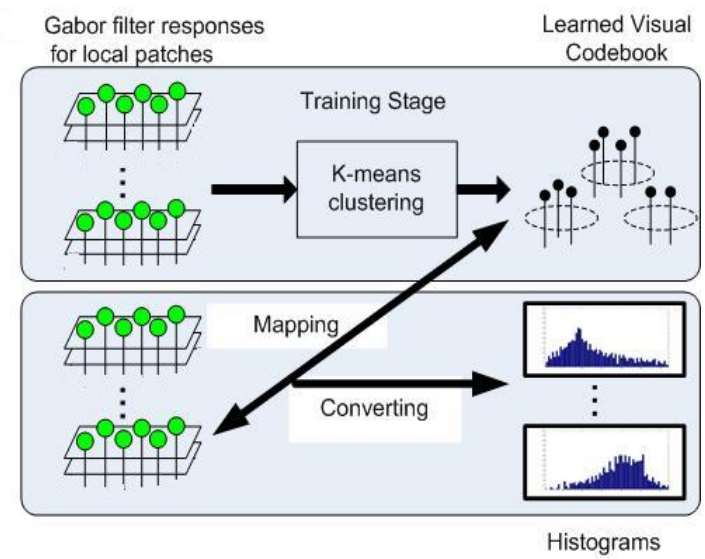

Fig. 2. The flowchart of LVC algorithm

\subsection{Eastern Code, Western Code and Human Code}

Learned Visual Codebook (LVC) has achieved good performance in 3D face recognition [5]. However, in this paper our task is 3D face ethnicity categorization. Intuitively, we first learn the visual codes for both eastern and western individuals, which are shown in Fig 3(a) Here, we adopt the Gabor features with only 1 scale and 2 orientations and QuadTree clustering to learn the visual codes [6]. From the figure, we find that there is a large overlap area between the eastern and western visual codes, which we can viewed as the common properties of humans (human codes). And there are also some codes corresponding to the properties of eastern or western individuals (eastern or western codes). Therefore, we can learn the eastern codes, western codes and human codes based on the obtained eastern and western visual codes.

Fig 3(b) shows three kinds of distance measures extracted from visual codes distribution. Because in LVC framework, each 3D face is divided into some local 


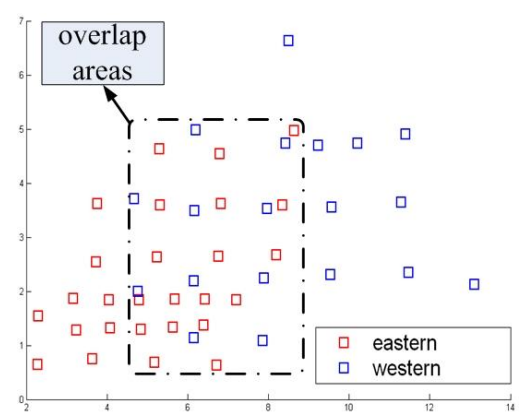

(a)

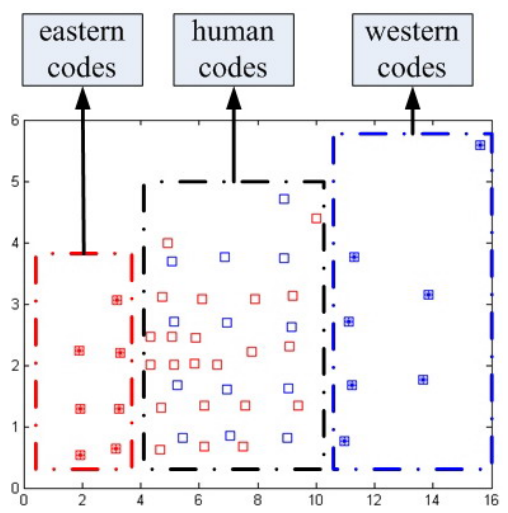

(c)

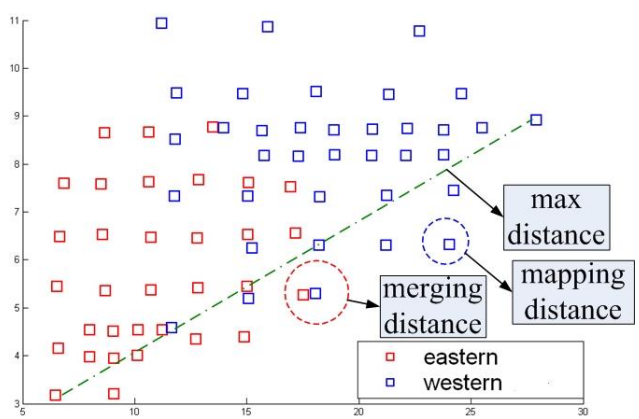

(b)

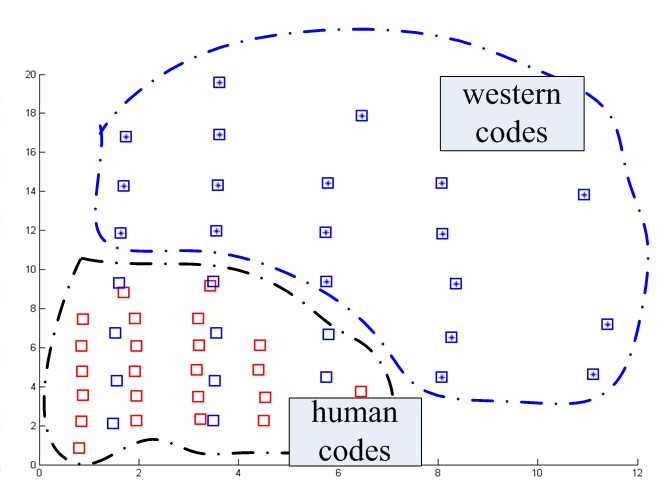

(d)

Fig. 3. How to learn the eastern, western and human codes. (a) is the centers of visual codes from both eastern and western individuals, (b) is the distance measures for the visual codes learning, (c) is the local patch with all three kinds of codes, (d) is the local patch without the eastern codes.

textures 5. Thus for each local texture, we compute their maximum distance between two visual codes (max distance). Then we can obtain the merging distance as follows:

$$
D 1=\operatorname{ratio} 1 * d \max
$$

where $D 1$ is the merging distance, ratiol is a predefined value and $d \max$ is the max distance. Merging distance is defined to generate eastern, western and human codes. When the distance between a western and an eastern visual code is below $D 1$, these two codes are categorized as the human codes. For a western visual code, when there is no eastern visual codes with the distance below $D 1$, this code can be categorized as western codes. For an eastern visual code, when there is no western visual codes with the distance below $D 1$, this code can be categorized as eastern codes. Some examples are shown in Fig $3(\mathrm{c})$ and Fig $3(\mathrm{~d})$ In some local textures, there will be no learned eastern codes. But there will be learned western codes for all local textures. 


\subsection{Membership Function}

Mapping distance is another distance measure shown in Fig 3(b) which is defined as

$$
D 2=\operatorname{ratio} 2 * d \max
$$

where $D 2$ is the mapping distance, ratio2 is a predefined value and dmax is the max distance. The definition of mapping distance is to compute the number of the Gabor feature vectors belonging to eastern and western categories, which is represented as eastnum and westnum. The initial values for eastnum and westnum are both zero. For each 3D face image, first we convert it to many Gabor feature vectors. Then if the distance between a vector and one of the eastern (western) codes is below mapping distance, the eastnum (westnum) adds one. The probability of the 3D face corresponding to eastern and western individuals are defined as follows:

$$
\begin{aligned}
& \operatorname{probability}_{\text {eastern }}(I)=\text { eastnum } / \text { num } \\
& \text { probability }_{\text {western }}(I)=\text { westnum } / \text { num }
\end{aligned}
$$

where $I$ is the input 3D face image, num is the total number of the Gabor feature vectors. Then our membership function is simply defined as

$$
\text { membership }(I)=\text { probability }_{\text {eastern }} / \text { probability }_{\text {western }}
$$

The obtained value is to represent the membership degree. When the value is small, the face image is similar to western individuals. And when the value is large, the face image is similar to eastern individuals.

\section{Experiments and Discussion}

Our proposed method is evaluated based on the FRGC2.0 3D Face Database and the expression division protocol proposed by Geometrix, which divides the FRGC2.0 data into three subsets: neutral, small expression and large expression [7] [8]. To achieve our ethnicity categorization task, we manually select the

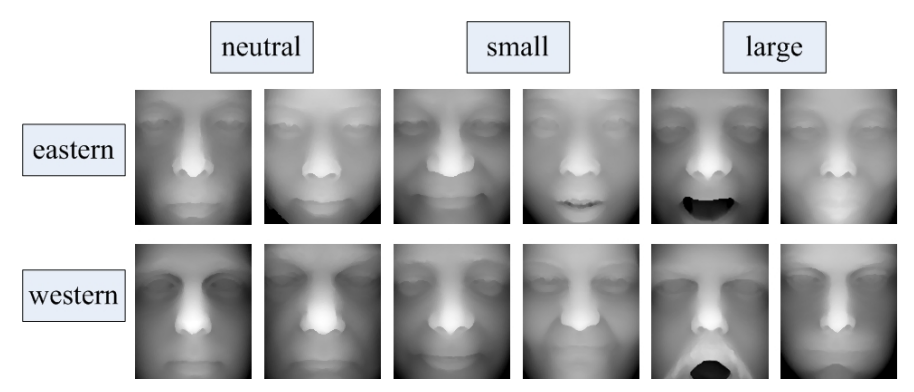

Fig. 4. Some example images in FRGC2.0 database (neutral subset). The first row shows the images of eastern people, the second row shows the images of western people. 
eastern individuals and western individuals for all these three subsets, in which we assign the ethnic categories such as Latin, Indian or African origins, into the western group. Then for the neutral expression subset, we have 807 eastern individuals and 1662 western individuals. For the small expression subset, we have 247 eastern individuals and 549 western individuals. For the large expression subset, we have 163 eastern individuals and 579 western individuals. Some example images are shown in Fig 4.

In our experiments, we only adopt 50 eastern individuals and 50 western individual with neutral expression as training set to learn the eastern codes, western codes and human codes. The testing stage contains three situations: neutral expression, small expression and large expression. The categorization performance is measured by the percentage of the eastern or western data located into the western, middle and eastern areas, which are shown in Table 1 and Table 2 . From Table 1, we find that the ratios of eastern individuals categorized into eastern area are $75.59 \%, 80.16 \%$ and $74.85 \%$ respectively in the three situations. From Table 2, we find that the ratios of western individuals categorized into western area are $89.65 \%, 79.05 \%$ and $77.20 \%$ respectively in the three situations.

Table 1. Eastern performance

\begin{tabular}{|c|c|c|c|}
\hline Category & Western & Middle & Eastern \\
\hline Neutral & $5.95 \%$ & $18.46 \%$ & $75.59 \%$ \\
\hline Small & $5.26 \%$ & $14.57 \%$ & $80.16 \%$ \\
\hline Large & $7.36 \%$ & $17.79 \%$ & $74.85 \%$ \\
\hline
\end{tabular}

Table 2. Western performance

\begin{tabular}{|c|c|c|c|}
\hline Category & Western & Middle & Eastern \\
\hline Neutral & $89.65 \%$ & $9.45 \%$ & $0.90 \%$ \\
\hline Small & $79.05 \%$ & $16.94 \%$ & $4.01 \%$ \\
\hline Large & $77.20 \%$ & $18.48 \%$ & $4.32 \%$ \\
\hline
\end{tabular}

\section{Discussion}

Experimental results illustrate our fuzzy 3D face categorization algorithm gives a comparable performance in all three data sets. Even in the large expression subset, more than $74 \%$ images are correctly categorized for both eastern and western individuals. Compared to previous methods, our method only adopt the simple Gabor features ( 1 scale and 2 orientations), no strong classifiers, such as SVM, Adaboost or Neural Network, are involved in the categorization procedures 9]. Therefore, our method is very promising and has great potential for the ethnicity categorization task, which is not limited in the 3D face modality.

From our experiments, we find that: First, only a small number of the individuals from Latin, Indian and African origins are mis-categorized. Because in data preparation section, we manually select these ethnic groups into the western individuals. Therefore, they have the western-similar faces. Second, although the western codes covers more space than the eastern codes. The distribution of the western individuals are more compact than the eastern individuals, as shown in Fig 5. In addition, the expression variations have little influence on the categorization performance for eastern individuals, while for western individuals, there is a distinct drop for the categorization performance when the situations change from neutral to small or large expressions. The main reason is that the data distribution of westnum is compact, but the data distribution of eastnum 


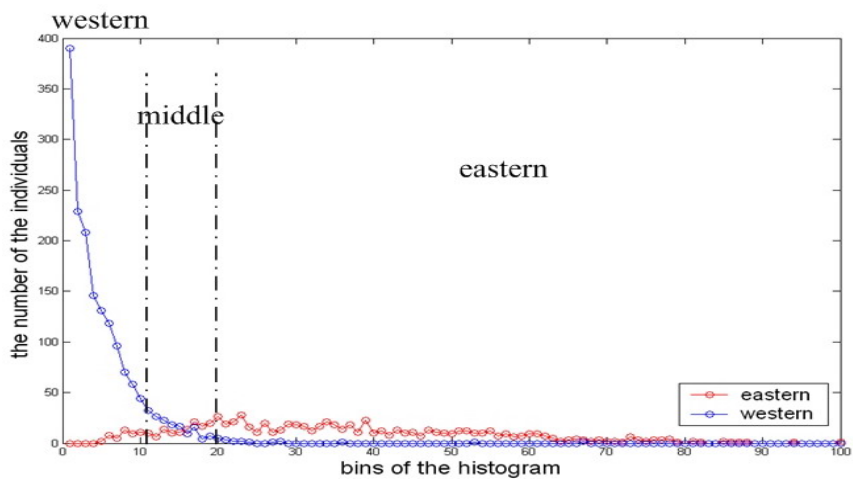

Fig. 5. The data distributions in the neutral subset

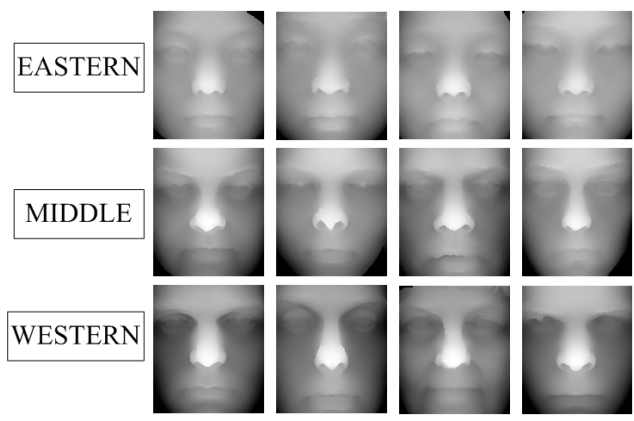

Fig. 6. The representative images in the categorized eastern, middle and western areas

covers a large area. This illustrates the appearances of western individuals are more similar and distribute tightly around some prominent facial structures, and the appearances of eastern individuals are more diversified and cover more smooth facial structures. Finally, from Fig [ we can find that the representative eastern individuals have a flat 3D face with no prominent structures, while the representative western individuals have a $3 \mathrm{D}$ face with sharp contrast and some prominent structures, such as the high-bridged nose and the deep-sunken eyes. The representative individuals in middle area are similar to the mixtures of the eastern and western individuals, which can be viewed as the transition between these two areas. Therefore, it is reasonable to use the fuzzy value for ethnicity categorization.

\section{Conclusion}

In this paper, we have proposed an efficient and accurate fuzzy 3D face categorization algorithm. Because we can learn the characteristics of the eastern and western individuals, for a $3 \mathrm{D}$ face, the membership degree can be obtained based 
on its similarity to the eastern or the western characteristics. Experimental results illustrate the effectiveness and robustness of our proposed fuzzy method for categorization.

\section{Acknowledgement}

This work is supported by the National Basic Research Program of China (Grant No. 2004CB318100), the National Natural Science Foundation of China (Grant No. 60736018, 60723005), the National Natural Science Foundation of China (Grant No. 60702024), the National Hi-Tech Research and Development Program of China (Grant No.2006AA01Z193, 2007AA01Z162) and the Chinese Academy of Sciences.

\section{References}

1. Zhao, W., Chellappa, R., Rosenfeld, A.: Face recognition: a literature survey. ACM Computing Surveys 35, 399-458 (2003)

2. Gutta, S., Wechsler, H., Phillips, P.J.: Gender and ethnic classification of face images. In: IEEE International Conference on Automatic Face and Gesture Recognition (1998)

3. Qiu, X., Sun, Z., Tan, T.: Global texture analysis of iris images for ethnic classification. In: Zhang, D., Jain, A.K. (eds.) ICB 2005. LNCS, vol. 3832, pp. 411-418. Springer, Heidelberg (2005)

4. Lu, X., Chen, H., Jain, A.K.: Multimodal facial gender and ethnicity identification. In: Zhang, D., Jain, A.K. (eds.) ICB 2006. LNCS, vol. 3832, pp. 554-561. Springer, Heidelberg (2006)

5. Zhong, C., Sun, Z., Tan, T.: Robust 3D face recognition using learned visual codebook. In: IEEE Conference on Computer Vision and Pattern Recognition (2007)

6. Zhong, C., Sun, Z., Tan, T.: Learning efficient codes for 3D face recognition. In: International Conference on Image Processing (2008)

7. Phillips, P.J., Flynn, P.J., Scruggs, T., Bowyer, K.W., Chang, J., Hoffman, K., Marques, J., Min, J., Worek, W.: Overview of the face recognition grand challenge. In: Proceedings of IEEE Conference on Computer Vision and Pattern Recognition (2005)

8. Maurer, T., Guigonis, D., Maslov, I., Pesenti, B., Tsaregorodtsev, A., West, D., Medioni, G.: Performance of geometrix activeid ${ }^{T M} 3 \mathrm{~d}$ face recognition engine on the frgc data. In: Proceedings of IEEE Conference on Computer Vision and Pattern Recognition (2005)

9. Duda, R.O., Hart, P.E., Stork, D.G.: Pattern Classification, 2nd edn. John Wiley \& Sons, Inc., Chichester (2001) 